\title{
The Supernova associated with GRB 030329
}

\author{
Shlomo Dado ${ }^{1}$, Arnon Dar ${ }^{1}$ and A. De Rújula ${ }^{2}$
}

\begin{abstract}
The relative proximity of the recent gamma ray burst (GRB) 030329 resulted in a large gamma-ray fluence and in the brightest-ever afterglow (AG), hours after the burst, in the radio, optical and X-ray bands, permitting precise AG measurements, sensitive tests of models and an excellent occasion to investigate the association of GRBs with supernovae (SNe). The Cannonball (CB) model provides a good, simple and universal description of all AGs of GRBs of known redshift, so that it is straightforward to use it to predict what the expected SN signatures are. In the case of GRB 030329, 10 days after burst the AG should begin to reveal the lightcurve, spectrum and polarization of an underlying SN — akin to SN1998bw - which will peak in the NIR/optical band around day 15. These effects will be easily observable if indeed SN1998bw is a good "standard candle" for GRB-associated SNe and if the so far unknown extinction in the host galaxy is not too large.
\end{abstract}

Subject headings: gamma rays: bursts

\section{Introduction}

It is important to know what it is that makes GRBs. The evidence for an association of long duration GRBs with supernovae (SNe) has been treated by observers with due care, since in only a few cases is the evidence very clear and model-independent. Moreover, the only overwhelmingly clear association — that of GRB 980425 with SN1998bw - does not fit at all in the generally accepted fireball models: the GRB is far too weak and the SN is annoyingly peculiar. These may be the reasons why authoritative reviews of the subject, even very recent ones, do not consider the SN/GRB association to be an established fact (e.g. Waxman 2003). To judge from recent Gamma Ray Burst Communication Network (GCN)

\footnotetext{
11dado@phep3.technion.ac.il, arnon@physics.technion.ac.il, dar@cern.ch. Physics Department and Space Research Institute, Technion, Haifa 32000, Israel

2alvaro.derujula@cern.ch. Theory Division, CERN, CH-1211 Geneva 23, Switzerland
} 
postings concerning GRB 030329, the observers' attitude is more positive: many GCNs discuss minor changes in the smooth afterglow (AG) decline as possible SN signatures, even much before - and a fluence level much larger - than one may expect them to occur.

In the CB model long-duration GRBs are assumed to be associated with common corecollapse SNe (Dar and De Rújula 2000). In this model there is nothing special about GRB 980425, which is describable in exactly the same way as all other GRBs of known redshift; nor is SN1998bw particularly peculiar: it did not emit the observed puzzling radio and X-ray signals, the CB responsible for the GRB did it, as discussed in tiresome detail in Dado el al. (2003a). It follows that, in the CB model, it makes sense to use SN1998bw as a putative standard candle for SNe associated with GRBs. The result of that exercise is that, in the $C B$ model, there is evidence for a GRB/SN association in all cases in which the SN could in practice be observed, about $1 / 2$ of the total (Dado et al. 2002a,b,c; 2003a,b,c).

The Cannonball (CB) model provides a good, simple and universal description of all AGs of GRBs of known redshift, $z$, so that it is straightforward to use it to predict what the expected SN contribution to the AG should be, since one is confident on the extrapolated CB contribution, a "background" to the SN signal. By making a fit to the observed AGs, before the SN contribution became observable, we have, in three cases, succesfully predicted what this contribution would be (Dado et al. 2002b,c, 2003b). We endeavour to do the same for GRB 030329, by submitting this paper to the astro/ph archives $\sim 2$ days before the SN contribution ought to become discernible. The credibility of our predictions is weakened by the fact that the data, so far preliminary and unpublished, are insufficient to estimate the extinction in the host galaxy. Also if - unlike in all other cases for which there is evidence of an association - the SN that generated GRB 030329 turns out to be more than two times less intrinsically luminous than SN1998bw, it will be difficult to see its contribution to the AG light curves, though its presence may still significantly distort the late-time AG spectrum.

We also show that -in the CB model- most of the "unusual" features of the AG of GRB 030329 occured before in other GRBs and could, in this sense, be anticipated. The early optical AG of GRB 030329 is, as for GRBs 990123, 021004 and 021121, a direct tracer of the expected circumburst density profile: in all these cases the AGs were "caught" very early and they provide extremely clean evidence for the expected $1 / r^{2}$ profile generated by a pre-SN stellar wind (Dado et al. 2003c). Both GRB 030329 and 021004 have $\gamma$-ray light curves dominated by two pulses or, in the CB model, two cannonballs. Both AGs display two wide shoulders that, in the same model, correspond to the contributions of the two CBs. The superimposed variations in the predicted smooth AG lightcurve, ups and downs of $\sim 1 / 2$ magnitude, are, as for GRBs 021004, 000301c and 970508, to be expected: they directly trace, like a needle on a vynil record, moderate deviations from a constant-density 
interstellar medium.

\section{GRB 030329}

The bright optical AG of this GRB (Vanderspek et al. 2003), which was first observed $\sim 1.25 \mathrm{~h}$ after burst by Peterson and Price (2003) and by Torii (2003) is - at magnitude $R \sim 12.5$ - by far the brightest optical afterglow observed hours after burst. The X-ray AG, also very bright, was seen at time $t \sim 5$ h after burst with RXTE, by Marshall and Swank (2003). The bright radio AG was observed at $t \sim 14 \mathrm{~h}$ by Berger et al. (2003). The redshift of the host galaxy was first determined by Greiner et al. (2003) to be $z=0.1685$. The large luminosity of this AG has triggered a large interest, reflected in a copious and enthousiastic release of GCN communications.

\section{The Cannonball Model of GRBs}

In the CB model (Dar and De Rújula 2000, 2001; Dado et al. 2002a; 2003a) reviewed in De Rújula 2002 and Dar 1993), long duration GRBs and their AGs are produced in corecollapse supernovae akin to SN1998bw by the ejection of bipolar jets of ordinary-matter, hydrogenic plasma clouds or "cannonballs" (CBs) with high Lorentz factors $\left(\gamma_{0} \sim 10^{3}\right)$. A $\mathrm{CB}$ is emitted, as observed in $\mu$-quasars, when part of an accretion disk falls abruptly onto the newly-born compact central object. Crossing the circumburst shells with a large $\gamma_{0}$, the surface of a $\mathrm{CB}$ is collisionally heated to $\mathrm{keV}$ temperatures and the thermal radiation it emits as it reaches the transparent outskirts of the shells - boosted and collimated by the CB's motion - is a single $\gamma$-ray pulse in a GRB. The cadence of pulses reflects the chaotic accretion and is not predictable, but the individual-pulse temporal and spectral properties are (Dar and De Rújula 2001; Dar 2003). In practice GRBs are observable only if the angle $\theta$ subtended by the CBs' velocity vector and the line of sight to the observer is small: $\theta=\mathcal{O}\left(1 / \gamma_{0}\right)$.

After becoming visible, a CB first cools by bremsstrahlung and expansion, emitting a hard spectrum that is seen in the X-ray band with a fluence decreasing with the expected $1 / t^{5}$ behaviour. When a CB's temperature approaches $\sim 1 \mathrm{eV}$ (within a few observer minutes), its emissivity is dominated by synchrotron emission from the electrons that penetrate in it as it propagates in the interstellar medium (ISM). Integrated over frequency, this synchrotron emissivity is proportional to the energy-deposition rate of the ISM electrons in the CB. These electrons are Fermi accelerated in the CB's tangled magnetic maze to a broken power- 
law energy distribution with a "bend" energy equal to their incident energy in the CBs' rest frame. Their synchrotron radiation - the afterglow - is also collimated and Doppler-boosted by the relativistic motion of the CBs. The radiation is also redshifted by the cosmological expansion and attenuated on its way to an earthly observer, during its passage through the CB itself, the host galaxy, the intergalactic space and our own galaxy.

\section{The NIR/Optical AG in the CB model}

In the CB model, the observed AGs have three origins: the ejected CBs, the concomitant SN explosion, and the host galaxy. These components are usually unresolved in the measured "GRB afterglows", so that the corresponding light curves and spectra are the cumulative energy flux density:

$$
F_{A G}=F_{C B s}+F_{S N}+F_{H G} .
$$

The contribution of the host galaxy, $F_{H G}$, is usually extracted from "very" late time observations when the $\mathrm{CB}$ and $\mathrm{SN}$ contributions become negligible, or is best fitted if such data are not available.

Let the energy flux density of SN1998bw at redshift $z_{b w}=0.0085$ (Galama et al. 1998) be $F_{b w}[\nu, t]$. For a similar SN placed at a redshift $z$ :

$$
\begin{aligned}
F_{S N}[\nu, t]= & \frac{1+z}{1+z_{b w}} \frac{D_{L}^{2}\left(z_{b w}\right)}{D_{L}^{2}(z)} \times \\
& F_{b w}\left[\nu \frac{1+z}{1+z_{b w}}, t \frac{1+z_{b w}}{1+z}\right] A_{S N}(\nu, z),
\end{aligned}
$$

where $A_{S N}(\nu, z)$ is the attenuation along the line of sight and $D_{L}(z)$ is the luminosity distance (we use a cosmology with $\Omega_{M}=0.3, \Omega_{\Lambda}=0.7$ and $H_{0}=65 \mathrm{~km} / \mathrm{s} / \mathrm{Mpc}$ ).

In the rest frame of a $\mathrm{CB}$ the $\mathrm{AG}$ that an observer sees at optical frequencies is given by:

$$
F_{C B}[\nu, t]=\frac{f[\gamma(t)]^{2} n_{p}}{\nu_{b}} \frac{\left[\nu / \nu_{b}\right]^{-1 / 2}}{\sqrt{1+\left[\nu / \nu_{b}\right]^{(p-1)}}},
$$

where $f$ is a normalization constant (see Dado et al. 2003a for its theoretical estimate), $\gamma(t)$ is the Lorentz factor of the $\mathrm{CB}, p \approx 2.2$ is the spectral index of the radiating electrons in the $\mathrm{CB}$, and $\nu_{b}$ is the "injection bend" frequency for an interstellar density $n_{p}$ :

$$
\nu_{b} \simeq 1.87 \times 10^{3}[\gamma(t)]^{3}\left[\frac{n_{p}}{10^{-3} \mathrm{~cm}^{3}}\right]^{1 / 2} H z .
$$


The theoretical motivation, as well as the excellent observational support for this "bend", are discussed in Dado et al. 2003a. An observer in the GRB progenitor's rest system, viewing a $\mathrm{CB}$ at an angle $\theta$, sees its radiation Doppler-boosted by a factor $\delta$ :

$$
\delta(t) \equiv \frac{1}{\gamma(t)(1-\beta(t) \cos \theta)} \simeq \frac{2 \gamma(t)}{1+\theta^{2} \gamma(t)^{2}}
$$

where the approximation is valid in the domain of interest for GRBs: large $\gamma$ and small $\theta$. The cannonballs' AG spectral energy density $F_{C B}^{o b s}$ seen by a cosmological observer at a redshift $z$ is:

$$
F_{C B}^{o b s}[\nu, t] \simeq \frac{A(\nu, t)(1+z) \delta(t)^{3}}{4 \pi D_{L}^{2}} F_{C B}\left[\frac{(1+z) \nu}{\delta(t)}, \frac{\delta(t) t}{1+z}\right]
$$

where $A(\nu, t)$ is the correction for the total extinction of the CB's radiation.

For an interstellar medium of constant baryon density $n_{p}$, the Lorentz factor $\gamma(t)$ is given by:

$$
\begin{aligned}
\gamma & =\gamma\left(\gamma_{0}, \theta, x_{\infty} ; t\right)=B^{-1}\left[\theta^{2}+C \theta^{4}+1 / C\right] \\
C & \equiv\left[2 /\left(B^{2}+2 \theta^{6}+B \sqrt{B^{2}+4 \theta^{6}}\right)\right]^{1 / 3} \\
B & \equiv 1 / \gamma_{0}^{3}+3 \theta^{2} / \gamma_{0}+6 c t /\left[(1+z) x_{\infty}\right]
\end{aligned}
$$

where $\gamma_{0}=\gamma(0)$, and $x_{\infty} \equiv N_{C B} /\left(\pi R_{\max }^{2} n_{p}\right)$ characterizes the CB's slow-down in terms of $N_{C B}$ : its baryon number, and $R_{\max }$ : its asymptotic radius (it takes a distance $x_{\infty} / \gamma_{0}$ for the $\mathrm{CB}$ to half its original Lorentz factor).

For AGs that are observed very early (those of the GRBs 021211, 990123, 021004 and 030329) the approximation of a constant ISM density, adopted above, is not good, since the CBs are first observed while travelling in the progenitor star's wind. The total wind grammage is insufficient to make $\gamma(t)$ deviate from $\gamma_{0}$ during the CB's traversal of the wind. Thus the only modification is that the density occuring in Eqs. (3) and (4) now becomes, for a typical "windy" profile:

$$
n(r)=n_{p}\left(1+r_{0}^{2} / r^{2}\right),
$$

where $n_{p}$ is again a constant. These fits have two extra parameters $\left(n_{p}\right.$, which was previously embedded with $f$, or played a very marginal role via Eq. (4), and $r_{0}$ ). Note that at very early time, $r \approx c t \gamma_{0} \delta_{0} /(1+z) \propto t$ and the above circumburst wind profile yields $F_{\nu} \propto$ $\left[1+(\bar{t} / t)^{2}\right]^{0.75}$ (Dado et al. 2003c), where $\bar{t}$ is the observer time when $r=r_{0}$ ). This very simple expression fits like the proverbial glove the data on the early time behaviour of the AGs of the GRBs 021211, 990123, 021004 and 030329, that in the fireball models would be attributed to a "reverse" shock. 
The contribution to $A(\nu, z)$ in Eq. (6) from selective extinction in the host galaxy and in the intergalactic medium can be estimated from the difference between the observed spectral index at very early time when the CBs are still near the $S N$ and that expected in the absence of extinction. Indeed, the $\mathrm{CB}$ model predicts - and the data confirm with precision - the gradual evolution of the effective optical spectral index towards the constant value $\approx-1.1$ observed in all "late" AGs (Dado et al. 2002a, 2003a). The "late" index is independent of the attenuation in the host galaxy, since at $t>1$ (observer's) days after the explosion, the CBs are typically already moving in the low column density, optically transparent halo of the host galaxy.

The comparison in Dado et al. (2002a,b,c; 2003a,b,c) of the predictions of Eq. (6) with the observations of optical, X-ray and radio light-curves and spectra for all GRBs of known redshift are very simple, satisfactory and parameter-thrifty.

\section{GRB 030329 in the CB model}

Six properties of AGs in the CB model are particularly relevant to GRB 030329:

- The optical AG is "caught" at very early times, when the CBs are still crossing the parent star's wind-generated density profile.

- In high-precission data, density inhomogeneities along the CBs' trajectory - expected within star formation regions and upon exit from the superbubbles where most SNe take place - result in observable achromatic "bumps" in the AG. These features have been seen in GRBs 970508, 000301c and 021004 (Dado et al. 2002a, 2003b).

- Except for GRB 021004, the past AG data were course enough or started late enough for the contributions of different CBs (which can often be resolved as individual pulses in the GRB phase) to coalesce into an AG describable by a single CB or a collection of similar ones. But individual CBs may have different properties, or be emitted at somewhat different angles, as observed in the $\mu$-quasar SS 433 (Margon 1984).

- The predicted AG spectra and their evolution, in particular the steepening at the timevarying frequency $\nu_{b}(t)$ of Eq.(4) towards $\nu^{-1.1}$ at late time, is very well supported by the data (Dado et al. 2002a, 2003a).

- The excess polarization of AGs above that induced by the ISM in the Galaxy may be largely due to the host galaxy's ISM. In that case, it should be correlated with the extinction in the host and decline with time as the CBs move into its halo. 
- The $\gamma$-ray light curve of GRB 030329, like that of GRB 021004, shows two prominent pulses (Vanderspek et al. 2003; http:// space.mit.edu/ HETE/ Bursts /GRB030329/).

In the CB model the two $\gamma$-ray pulses of the last item correspond to two dominant CBs. The good quality and early start of the optical data for GRB 030329 implies that the individual contributions of the two CBs are discernable in the AG: we must fit the broadband AG light curves with the additive contributions of two CBs, emitted almost in the same direction, but with otherwise free parameters (normalization, $\gamma_{0}$ and $x_{\infty}$ ). We fix the spectral index $p$ in Eq. (3) to the theoretically expected $p=2.2$, assume the density profile given by Eq. (8), and fit simultaneously all the reported NIR, optical and radio data.

The individual CBs' contribution are given by Eq. (6) which implicitely uses all equations from to (3) to (7). The free parameters are the radius $r_{0}$ at which the wind and ISM densities

are equal and, for each $\mathrm{CB}$, the viewing angle, $\theta$, the normalization constant $f \times n_{p}$, the initial Lorentz factor, $\gamma(0)$ and the decelaration parameter, $x_{\infty}$.

The $R$-band magnitude of a SN akin to $1998 \mathrm{bw}$, displaced to $z=0.1685$, is $R \sim 20.2$ at peak brightness. Thus, in our fits, we neglect the smaller contribution of the host galaxy, $R>23.1$ at $2 \sigma$ confidence level (Blake and Bloom, 2003; see also Wood-Vasey et al. 2003).

\subsection{NIR-Optical AG}

In Fig. 1 we show the CB-model's fit to data for the NIR-optical light-curves reported in the GCN circulars 1990, 2001, 2024, 2046, 2051, 2054, 2079 (Burenin et al.); 1991, 2005, 2028 (Rumyantsev et al.); 1992 (Andersen et al.); 1995 (Rykoff et al.); 1999 (Gal-Yam et al.); 2002, 2035, 2091 (Lipunov et al.); 2012 (Martini et al.); 2016 (Masi et al.); 2019 (Smith et al.); 2021 (Halpern et al.); 2022, 2075 (Zharikov et al.); 2029 (Klose et al.); 2030 (Bartolini et al.); 2034, 2045, 2049, 2060 (Lipkin et al.); 2036 (Garnavich et al.); 2037 (Moran et al.); 2040, 2096 (Lamb et al.); 2041 (Stanek et al.); 2042 (Schaefer. et al.); 2048 (Zeh et al.) 2056, 2065, 2070 (Fitzgerald \& Orosz); 2063 (Weidong Li et al.); 2066 (Tober et al.); 2050, 2067, 2083, 2097 (Pavlenko et al.); 2068 (Bloom et al); 2074 (Cantiello et al.); 2077, 2084 (Ibrahimov et al.); 2080 (Sato et al.); 2094 (Khamitov et al.); 2096 (Lee et al.); and 2098 (Ibrahimov et al.).

All the observational data was recalibrated to the field photometry of Henden et al. (2003), either by the observers or by ourselves. We have corrected for selective extinction in our galaxy (Schlegel et al. 1998): $E(B-V)=0.025$ in the direction of GRB 030329. We did not correct for extinction in the host galaxy; the preliminary data in the GCN does not provide clear evidence for it. 
The broad-band fitted parameters of the two CBs are $\theta[1]=2.00 \mathrm{mrad}$, a nearly identical $\theta[2]=1.95 \mathrm{mrad}$ for the second CB's direction, $\gamma_{0}[1]=1477, \gamma_{0}[2]=976$ (implying $\delta_{0}[1]=$ $\left.306, \delta_{0}[2]=423\right), \mathrm{x}_{\infty}[1]=476 \mathrm{kpc}$, and $\mathrm{x}_{\infty}[2]=36 \mathrm{kpc}$. The density and "wind" parameters of Eq. (8) are $n_{p}=0.86 \times 10^{-2} \mathrm{~cm}^{-3}$ and $r_{0}=26 \mathrm{pc}$, which yield $\rho r^{2}=9.3 \times 10^{13} \mathrm{~g} \mathrm{~cm}^{-1}$ for the progenitor-wind's grammage, similar to the canonical value for the winds of the parent massive stars of core-collapse SNe (Dado et al. 2003c and references therein).

To demonstrate the real quality of the fit, we have blown up the R-band results in Fig. 2. In the region between $t \sim 1$ and $\sim 5$ days, the data "wiggles" by as much as $30 \%$ around the smoother theoretical curve. It would be easy to correct for this by assuming similar deviations of the ISM density input in Eqs. (3) and (8), relative to a constant $n_{p}$, clearly a moot exercise.

A SN1998bw-like contribution, as can be seen in Figs. 1 and 2, will be observable. Since the contribution of the second CB is still considerable near the peak brightness of the SN (at $t \sim 15$ days) the SN contribution appears as a shoulder in the light curves. If this contribution is much fainter - due to extinction in the host galaxy or a possible deviation from a so-farsuccesful SN1998bw "standard candle" ansatz - its presence could still be established by the change of colours from the broad band $F_{\nu} \sim \nu^{-1.1}$ behaviour of the CBs' emission towards $F_{\nu} \sim \nu^{-3.5 \pm 1.0}$, the SN spectrum.

\subsection{Optical Polarization}

In the $\mathrm{CB}$ model, after subtraction of the Galaxy's contribution, the polarization of the optical AG is mainly due to the effect of the host's ISM. It is therefore correlated with the extinction in the host, has a fixed angular position, and diminishes with time as the CBs escape into the galactic halo. Consequently near peak brightness of the associated SN (around 15 days after burst) the polarization of the AG should be that of the underlying SN.

\subsection{The GRB proper}

In the $\mathrm{CB}$ model, due to Doppler boosting and relativistic collimation (Dar and De Rújula 2000), the $\gamma$-ray fluence of a GRB viewed at a small $\theta$ is amplified by a huge factor $\delta_{0}^{3}$.

$$
F_{G R B}=\frac{(1+z) \delta_{0}^{3}}{4 \pi D_{L}^{2}} E_{\gamma}
$$


relative to $E_{\gamma}$, the total energy in photons emitted by the CBs in their rest system. The total "equivalent spherical", or would-be isotropic energy, $E^{i s o}$, inferred from the observed fluence, is a factor $\left(\delta_{0}\right)^{3}$ larger than $E_{\gamma}$. In Dado et al. 2002a we deduced that the $E_{\gamma}$ values of the GRBs of known $z$ span the surprisingly narrow ${ }^{3}$ interval $10^{44 \pm 0.3} \mathrm{erg}$, the spread in $F_{G R B}$ being mainly due to the spread in their values of $\delta_{0}$ (deduced from the fits to their AGs). For GRB 030329 the CB-model fit to its broad band AG yields $\delta_{0}[1]^{3}=2.86 \times 10^{7}$ and $\delta_{0}[2]^{3}=7.56 \times 10^{7}$. The CB-model expectation from the fitted AG is $E^{i s o} \approx\left(\delta_{0}[1]^{3}+\right.$ $\left.\delta_{0}[1]^{3}\right) E_{\gamma} \approx 1.04 \times 10^{52 \pm 0.3} \mathrm{erg}$, in agreement with the observed $E^{i s o} \approx 1.1 \times 10^{52} \mathrm{erg}$, deduced from its measured redshift, $z=0.168$, and fluence in the $30-400 \mathrm{keV}$ band $\sim 10^{-4} \mathrm{erg} \mathrm{cm}^{-2}$ (Vanderspek et al. 2003).

At the very early stage of $\gamma$-ray emission, CBs are still dense enough for the ambient protons to interact hadronically with those of the CB's ordinary-matter plasma. In the simplest "surface model" of the generation of the GRB proper, the energy deposited in the CBs by these interactions is reemitted with a quasi-thermal spectrum with a predictable timedependent temperature, leading to the correct predictions of the very stable characteristic energy of GRBs, and of the observed spectral indices of their "Band" spectrum (Dar and De Rújula 2000, Dar 2003).

The light curve of a GRB's single pulse rises abruptly as the column density of the material in front of a $\mathrm{CB}$ descends to $\mathcal{O}(1)$ attenuation lengths, and diminishes thereafter as the density of the material the $\mathrm{CB}$ encounters diminishes with time. For a windy $1 / r^{2}$ density profile of this density, the shape of a pulse at fixed energy is analytical and simple:

$$
\begin{aligned}
F_{C B}(t) & \propto \frac{1}{t^{2}} \operatorname{Exp}\left[-2 \frac{\Delta t}{t}\right] \\
\Delta t & =\frac{\Delta x(1+z)}{c \gamma_{0} \delta_{0}},
\end{aligned}
$$

with $t$ the observer's time and $\Delta x$ the proper distance travelled by the CB from the onset of the pulse to the position at which one $\gamma$-ray absorption length is still in front of it. In the rough approximation of an energy-independent $\gamma$-ray attenuation in the circumburst material, the shape of an energy-integrated pulse is the same as in Eq. (10).

We have constructed a rough description of the $\gamma$-ray light curve of GRB 030329 (Vanderspek et al. 2003; http:// space.mit.edu/ HETE/ Bursts /GRB030329/) by summing two pulses, both with the shape of Eq. (10), separated by 12 observer's seconds, and with $\Delta t[1]=6 \mathrm{~s}, \Delta t[2]=4 \mathrm{~s}$. The result is shown in Fig. 3. The description of the GRB is quite

\footnotetext{
${ }^{3}$ GRBs in the CB model are much better standard candles than in the standard model (Frail et al. 2001).
} 
satisfactory, even though we would have expected $\Delta t[1] / \Delta t[2]=\gamma_{0}[2] \delta_{0}[2] /\left(\gamma_{0}[1] \delta_{0}[1]\right) \sim 1.1$, as opposed to 1.5. It would be easy to find excuses to exit from this minor problem (e.g. we have not quoted errors in our parameters, that are made somewhat meaningless by the slightly erratic behaviour of the AG light curves).

\section{Conclusions}

We have shown that the data on GRB 030329 and on its early NIR-optical AG are well described by the CB model. This is also the case for all other GRBs of known redshift, including GRB 980425, whose associated SN we use as a putative standard candle.

In the CB model, we contend, the association between long-duration GRBs and SNe is extremely well established: in all GRBs of known redshift (all those with $z<1.12$ ) a SN1998bw-like supernova could be seen, and with various degrees of significance, it was seen. SN1998 stood the "standard-candle" test with surprising precision. In many cases, though, the test depended on very significant extinction corrections in the host galaxy. If this extinction turns out to be significant for GRB 030329, the direct observation of a SN signal in the AG could be jeopardized. In such a case one would need precise colour photometry and spectroscopy with the most powerful telescopes, such as VLT, Magellan, Keck and HST, and very late time measurements of the host galaxy's magnitude.

Acknowledgment: This research was supported in part by the Helen Asher Space Research Fund at the Technion. Arnon Dar thanks the TH division at CERN for its hospitality.

\section{REFERENCES}

Andersen M. I. et al. 2003, GCN Circ. 1992

Bartolini, C., et al. 2003, GCN Circ. 2030

Berger, E. et al. 2003, GCN Circ. 2014

Blake, C. \& Bloom, J. S., GCN Circ. 2011

Bloom, J. S., et al. 2003, GCN Circ.2068

Burenin, R., et al. 2003, GCN Circs. 1990, 2001, 2024, 2046, 2051, 2054, 2079

Cantiello, M., et al. 2003, GCN Circ.2074 
Dado, S., Dar A. \& De Rújula, A. 2002a, A\&A, 388, 1079

Dado S., Dar A., De Rújula A., 2002b, ApJ, 572, L143

Dado S., Dar A., De Rújula A., 2002c, A\&A, 393, L25

Dado S., Dar A., De Rújula A., 2003a, A\&A, 401, 243

Dado S., Dar A., De Rújula A., 2003b, ApJ, 585, L15

Dado S., Dar A., De Rújula A., 2003c, astro-ph/0302429

Dar, A. \& De Rújula, A. 2000, astro-ph/0008474

Dar, A. \& De Rújula, A. 2001, astro-ph/0012227

Dar, A. 2003, astro-ph/0301389

De Rújula, A. 2002, astro-ph/0207033

Fitzgerald, J. B., \& Orosz, J. A., 2003, GCN Circs. 2056, 2065, 2070

Galama T.J., et al., 1998, Nature 395, 670

Gal-Yam, A., et al. 2003, GCN Circ. 1999

Garnavich, P., et al. 2003, GCN Circ. 2036

Greiner, J., et al. 2003, GCN Circ. 2020

Halpern J. P., et al. 2003, GCN Circ. 2021

Henden, A. A., et al. 2003, GCN Circ. 2082

Ibrahimov, M. A., et al. 2003, GCN Circs. 2077, 2084

Khamitov, I., et al. 2003, GCN Circ. 2094

Klose, S., et al. 2003, GCN Circ. 2029

Lamb D. Q., et al. 2003, GCN Circs. 2040, 2096

Lee, B. C., et al. 2003, GCN Circ. 2096

Li, W., Filippenko, A. V,, Chornock, R. \& Jha, S. 2003, ApJ, 586, L9

Li, W., et al. 2003, GCN Circ. 2063 
Lipkin, Y., et al. 2003, GCN Circs. 2034, 2045, 2049, 2060

Lipunov, V., et al. 2003, GCN Circs. 2002, 2035, 2091

Margon, B.A., 1984, ARA\&A, 22, 507

Marshall, F. E. \& Swank, J. H. 2003, GCN Circ. 1996

P. Martini, P., et al. 2003, GCN Circ. 2012

Masi, G., et al. 2003, GCN Circ. 2016

Moran, J., et al. 2003, GCN Circ. 2037

Pavlenko, E., et al. GCN Circs. 2050, 2067, 2083, 2097

Peterson, B. A. \& and Price, P. A. 2003, GCN Circ. 1985

Rumyantsev, V., et al. 2003, GCN Circs. 1991, 2005, 2028

Rykoff, E. S., et al. 2003, GCN Circ. 1995

Sato, R., et al. 2003, GCN Circ. 2080

Schaefer, J., et al. 2003, GCN Circ. 2042

Schlegel D. J., Finkbeiner D. P. \& Davis M. 1998, ApJ, 500, 525

Smith, D. A., et al. 2003, GCN Circ. 2019

Stanek, K. Z., et al. 2003, GCN Circ. 2041

Tober, J., et al. 2003, GCN Circ. 2066

Torii, K. 2003, GCN Circ. 1986

Vanderspek, V., et al. 2003, GCN Circ. 1997

Waxman, E., 2003, astro-ph/030351

Zeh, A., et al. 2003, GCN Circ. 2048

Zharikov, S., et al. 2003, GCN Circs. 2022, 2075 


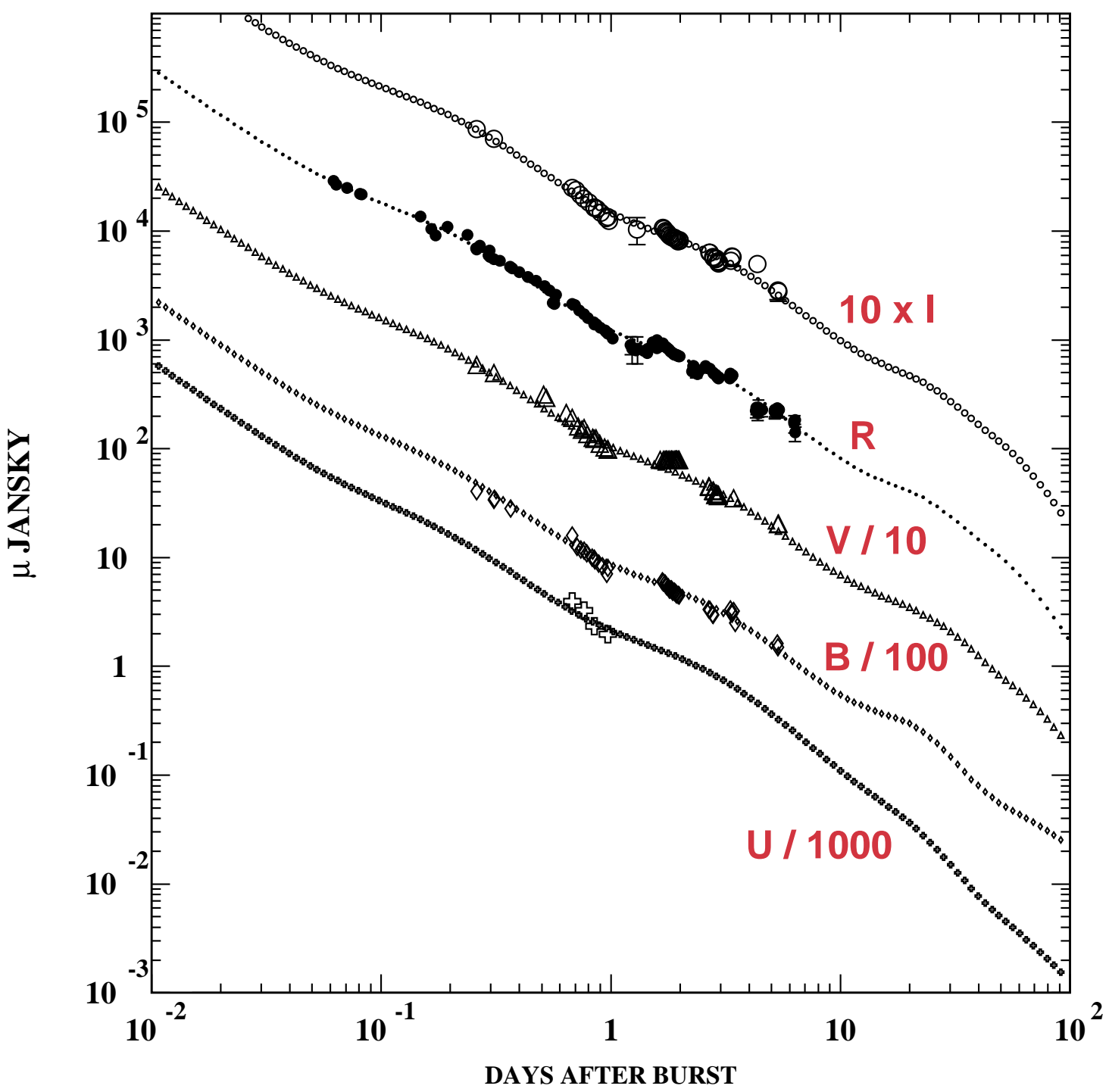

Fig. 1. - The NIR-optical observations of the AG of GRB 030329 and the fit for two CBs with different parameters, as described in the text. The ISM density is a constant plus a "wind" contribution decreasing as $1 / r^{2}$. The various bands are scaled for presentation. The data was selected from the GCN notices quoted in the text, recalibrated with the observations of Henden et al. 2003. The host-galaxy's contribution was neglected. The individual bands have been rescaled for clarity. 


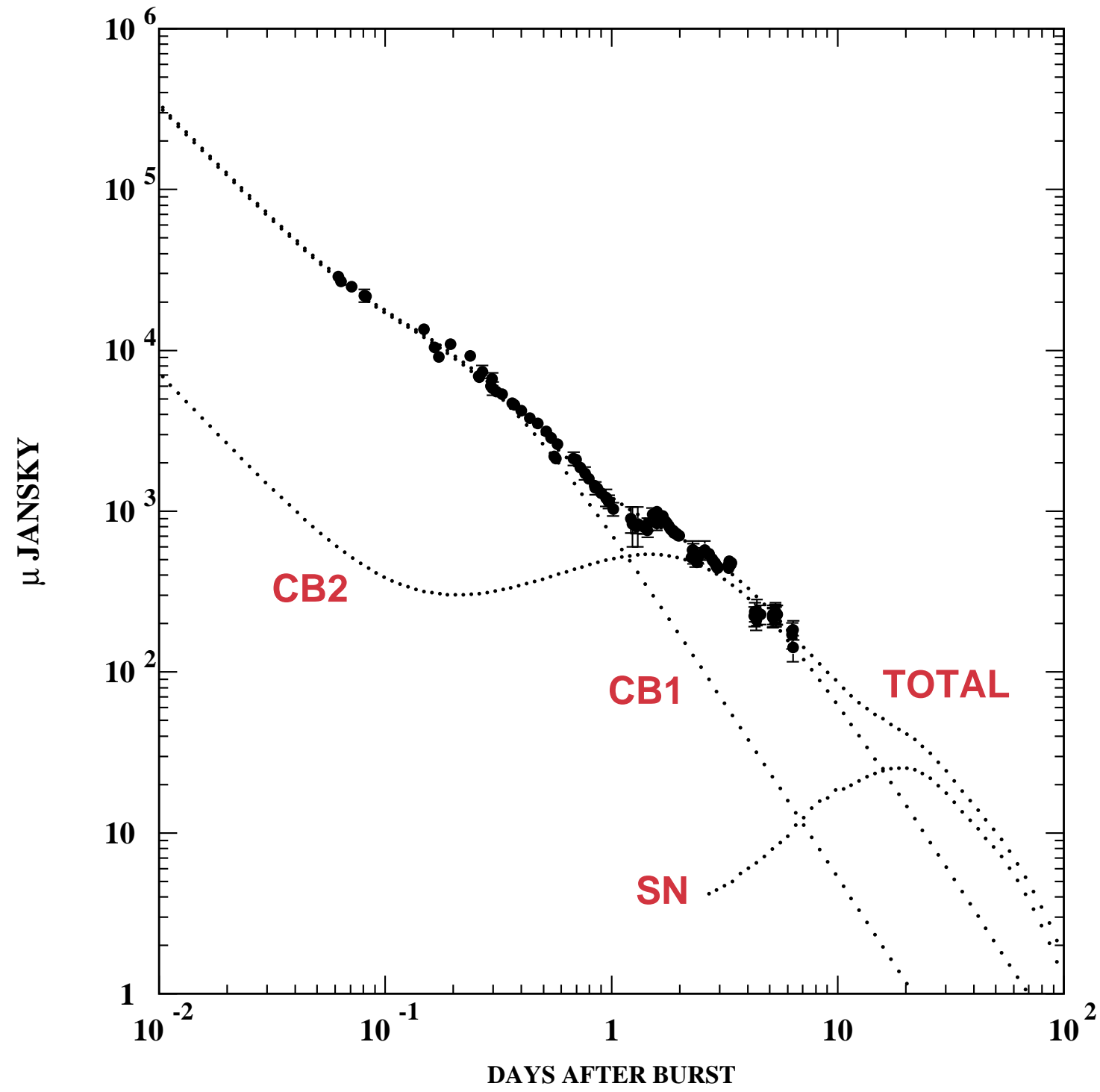

Fig. 2.- Blow-up of the R-band results of Fig. 1. The ISM density was assumed to be a constant plus an additional "wind" contribution decreasing as $1 / r^{2}$. The wind contribution is only significant at $\mathrm{t}<0.1$ days, after which the CBs are more than $10 \mathrm{pc}$ away from the progenitor. This "wind" is also seen in other AGs observed early enough (Dado et al. 2003c). The individual contributions of the two CBs and of a SN akin to SN1998bw (at the GRB's redshift) are also shown. 


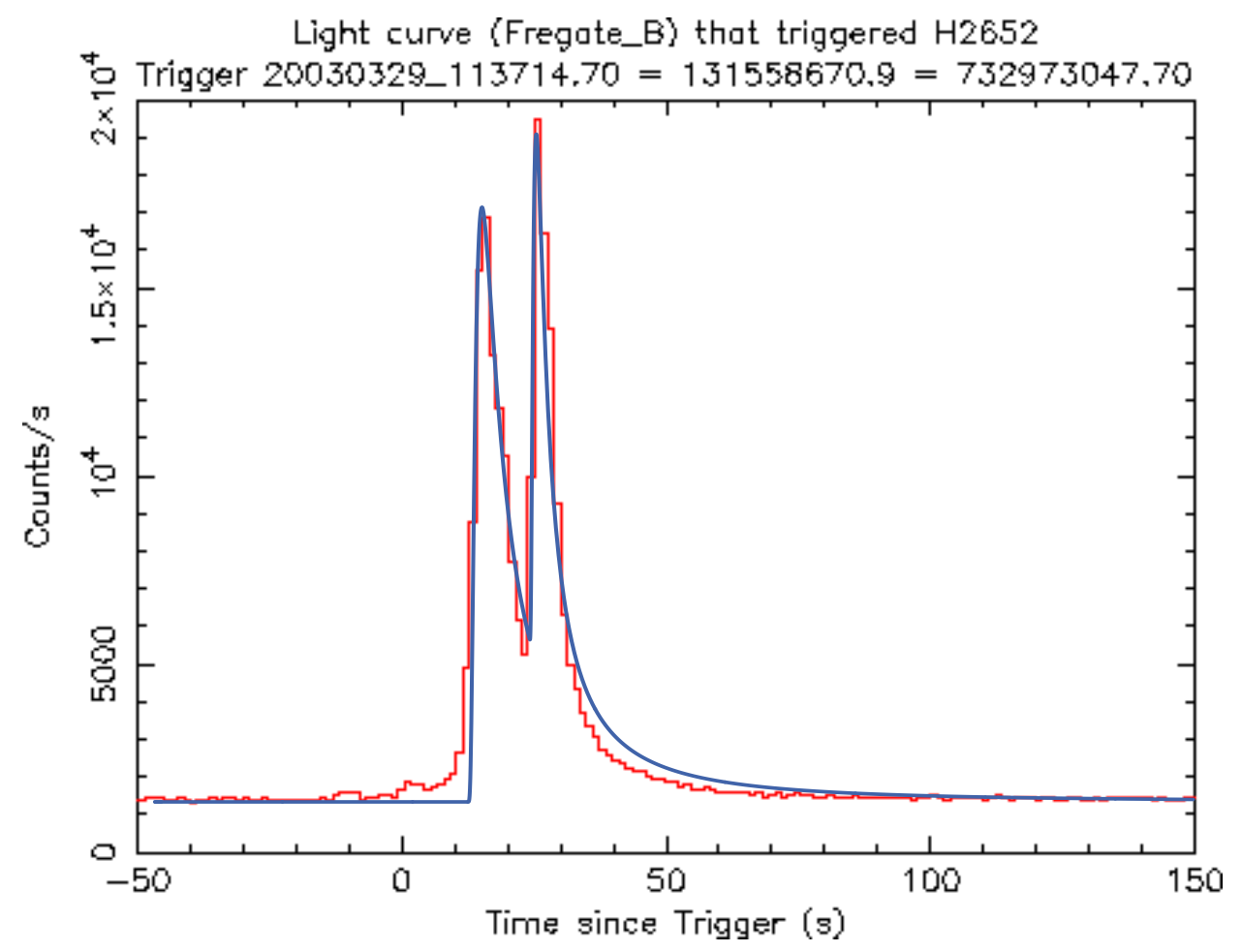

Fig. 3.- The $\gamma$-ray light curve of GRB 030329 (the red binned curve), and its simple CB-model description (the blue continuous line). 